\title{
Image Based Group Classifier for Brain Tumor Detection Using Machine Learning Technique
}

\author{
Putta Rama Krishnaveni*, Gattim Naveen Kishore
}

ECE Department, KLEF Green Fields, Vaddeswaram 522502, Guntur District, Andhra Pradesh, India

Corresponding Author Email: rkrishnaveni41 @ gmail.com

https://doi.org/10.18280/ts.370520

Received: 9 July 2020

Accepted: 22 September 2020

\section{Keywords:}

malignant tumor, feature extraction, classification, segmentation

\begin{abstract}
In view of insights of the Central Brain Tumor Registry of the United States (CBTRUS), brain tumor is one of the main sources of disease related deaths in the World. It is the subsequent reason for tumor related deaths in adults under the age 20-39. Magnetic Resonance Imaging (MRI) is assuming a significant job in the examination of neuroscience for contemplating brain images. The investigation of brain MRI Images is useful in brain tumor analysis process. Features will be extricated and selected from the segmented pictures and afterward grouped by utilizing the classification procedures to analyze whether the patient is ordinary (having no tumor) or irregular (having tumor). One of the most dangerous cancers is brain tumor or cancer which affects the human body's main nervous system. Infection that can affect is very sensitive to the brain. Two types of brain tumors are present. The tumor may be categorized as benign and malignant. The benign tumor represents a change in the shape and structure of the cells, but cannot contaminate or spread to other cells in the brain. The malignant tumor can spread and grow if not carefully treated and removed. The detection of brain tumors is a difficult and sensitive task involving the classifier's experience. In the proposed work a Group based Classifier for Brain Tumor Recognition (GbCBTD) is introduced for the efficient segmentation of MRI images and for identification of tumor. The use of Convolutional Neural Network (CNN) system to classify the brain tumor type is presented in this work. Relevant features are extracted from images and by using CNN with machine learning technique, tumor can be recognized. CNN can reduce the cost and increase the performance of brain tumor detection. The proposed work is compared to the traditional methods and the results show that the proposed method is effective in detecting tumors.
\end{abstract}

\section{INTRODUCTION}

The brain tumor is one of the main cancer-related causes of death in India, based on statistics from the Central Brain Tumors Registry. Leukemia is the second most important cause of cancer death in children below 20 and in men between 20-39 years. It is the first cause of cancer death in children under 20. Each year, an estimated 87,000 adults, including malignant $(37,000)$ and non-malignant $(50,000)$ in India, receive a new brain tumor diagnosis [1]. An estimated 21,000 deaths are attributed to malignant brain tumor, 6700 of which have been diagnosed in children less than 15 [2].

The most common use of medical images is to visualize the inner part of the human body [3]. They are crucial if serious diseases are to be detected and treated [4]. Magnetic Resonance Imaging is one of the most commonly used methods of medical imaging [5]. The patient's MRI is in a machine producing a powerful magnetic field to match the protons [6]. The alignment is then broken by sending a pulse of the radio frequency [7]. The protons are adjusted to the magnetic field when the pulse radiofrequency ends. The time required for the reconfiguration and energy released during this event varies depending on the tissue [8]. A 3D image MRI is built by recording this information. Figure 1 depicts the brain tumor identified image.

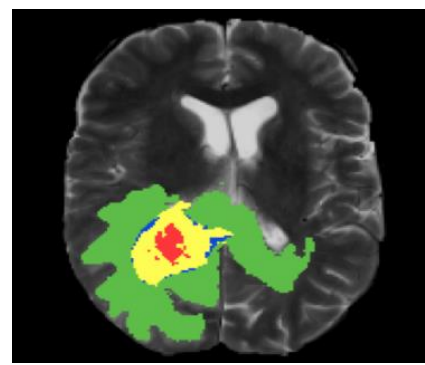

Figure 1. Brain tumor identified image

Supervised segmentation Methods are controlled and involve segmentation feedback and user interaction. The user needs to mark the region or provide the algorithm with the correct performance of the segmentation [9]. The classification based method, which is the main scope of this work, is an example of a workable method. Supervised Segmented Image classification shall label an image or part with the corresponding classes based on its features. Two main steps lie in the classical approach to classification [10]. The first is the extraction of features. Important information is obtained in this step on the class labels [11]. For these features, edges, corners and color data are just a few examples. The second step is to determine the class of the features [12]. This step is done with machine learning. Equipment is indeed high- 
dimensional vectors that live in an object-content area. Machine learning algorithms attempt to divide the function space into meaningful, class-related regions [13]. If an object's feature vector is in one of these regions, the corresponding class is foreseen. Supervised and unattended learning can be used to train the machine learning algorithms [14].

The segmentation of images is to divide an image into its consistent components [15]. These partitions have similar characteristics after segmentation like color, texture, etc. [16]. The most common methods used in the literary segmentation of images include clustering, edge-based, compression-based methods, region-based methods, pixel-based methods, and graph-based methods [17]. Each pixel is assigned a class label in semantic segmentation, and when these pixels are combined they fit the segments in the image [18]. Image segmentation can be classified as unattended and controlled segmentation. Uncontrolled segmentation methods provide input, but the user does not provide basic details for algorithm training or user interaction. Therefore, the algorithm itself knows or does not need to be tracked [19].

Early detection of brain tumors can therefore play a crucial role in improving treatment options and increasing the likelihood of survival [20]. However, the time-consuming, complex, and heavy process of manual segmentation is the source of many images of MRIs in medical routine [21]. MRI is primarily used for identifying brain tumors or lesions, also known as Magnetic Resonance Imaging [22]. MRI brain tumor segmentation is one of the most critical tasks in medical imaging, since a large number of data is normally involved. In addition, soft tissue boundaries can be unchanged for the tumors. It is therefore a very detailed process to correctly classify the tumors of the human brain [23].

In the proposed work, an image based group classifier is designed for the accurate detection of brain tumor using the machine learning techniques. The proposed model segments the brain image and then perform pixel extraction from each segment. The designed classifier considers only relevant features for the detection of brain tumor. The Image based group classifier performs generation of clusters which holds relevant features of a group in a single cluster and then the classifier uses these features for the prediction of brain tumor. The rest of the paper is organized as section 2 gives a brief survey on the traditional brain tumor detection models and the section 3 describes the proposed model and its process of identification of brain tumor. The section 4 illustrates the results obtained which are compared with the traditional models and finally section 5 concludes the papers.

\section{LITERATURE SURVEY}

Brain tumor is the abnormal growth of cells in the brain, discussed by Qian et al. [1]. Two major cancers such as benign and malignant tumors exist. The primary and secondary tumor is usually known as tumor. The primary tumor begins in the brain and the secondary tumor spreads to other body sections. Many different types of medical imaging such as X-ray, CT (Computed Tomography) and MRI (Magnetic resonance imaging) are available. Due to the high resolution and decent image quality, the model uses MRI brain pictures. The tumor area needs to be removed from the MRI brain image after captured. The radiologist helps to prepare correct segmentation of medical images. Astrocytoma is the most common type of tumor. The study primarily separated the brain tumor glioma types.

The image threshold technique is based on the local imaging properties, where the threshold value is modified in a specific image region, depending on the intensity distribution. This is useful if the whole picture does not fit or needs to be standardized separately for various sections of the picture, e.g. due to image homogeneity. Havaei et al. [2] used this method for the segmentation of brain tissue. Generally, thresholdbased techniques are useful if there is a high gap between objects and backgrounds, not brain MR pictures. In addition, the threshold calculation is typically challenging and involves contact with the user. They may however be used as an initial step in the segmentation process.

Menze et al. [3] have taken a different approach in order to prevent over-segmentation, rather than post-processing, on the basis of regional fusion, input images. It included reducing noise, attenuating the curvilinear structures present in MR images, and calculating the morphological gradients and marks of the input image. The findings reported were not satisfactory. The segmentation algorithm for glioma-based brain tumors was suggested by Chaurasia and Culurciello [4]. The tumor has an irregular shape that is found using a deep convolutionary neural network algorithm. Thus, the patient's survival rate is improved by specific brain tumor segmentation. By entering max-out and lowering layers in the patch processing, the issue of overfitting is eliminated. The proposed algorithm also uses a pre-processing method to eliminate undesirable noise and to remove small false positive noise from the morphological operators.

Bauer et al. [5] recommends the up-to - date output of the convolutionary neural network for automatic fragmentation of health data. However, the findings for clinical use are not robust. It limits the generality of previously unknown classes of objects. The problem is remedied by a modern interactive segmentation system based on a deep learning by connecting CNN to a bounding box and scripting segmentation pipeline. The approach proposed makes the CNN model adaptable to a particular test picture that can be unmonitored or controlled.

A widespread master education technique has been provided by Qian et al. [6] to identify and segment medical images. In this method, convnet is used to distinguish medical brain images into healthy and unhealthy brain images. The approach used classifies the brain tumor as low and large. The MRI brain tumors are classified through the Alex Krizhousky network deep learning architecture. The classification of tumors takes place on the entire picture instead of pixels.

Xia et al. [9] has established a brain lesion segmentation, which is a difficult process, using the 3-D neural convolution network. In order to extract the local and larger contextual details, the design of the dual pathway operates an image on many scales. The false positives that have been deleted with a completely connected $3 \mathrm{D}$ random conditional region. The method of segmentation was used for multi-channel MRI lesions of traumatic brain injury, brain tumors and ischemic stroke. The 3D CNN is an efficient way of dividing without raising the cost and number of training parameters into good segmentation.

Jafari et al. [15] implemented the Learning Vector Quantization approach based on a probabilistic neural network model. The model was tested on 95 MRI images, including 25 MRI images, and the remainder was used as a training session. The photos were flattered by the Gaussian filter. The updated system reduced 67 percent of the processing time. The MRI images are transformed to matrices, which are then defined by 
the Probabilistic Neural Network. Quality analysis is eventually carried out. There were 20 subjects in the preparation and fifteen subjects in the research dataset. Accuracy was between $70 \%$ and $85 \%$ based on the spread value.

Parihar [19] performed an evaluation of the efficiency of an interactive three dimensional image segmentation technique. They demonstrated improved time and precision of contact in the subject over the contouring of the hand. In their study, deficiencies were found in areas where the boundaries were defined in the data and the awareness of using segmental experts to define fundamental truth was raised. Oversegmentation is the issue of the waterfront.

\section{PROPOSED MODEL}

The main idea of the proposed model is to divide and diagnose brain tumor by means of an algorithm of machine learning and to determine the classification criteria for our model. The proposed brain image segmentation system involves different stages; skull striping, filtering and updating, segmentation; morphological operations; tumor contouring. The advantages of brain visualization have also provided large volumes of data of higher quality [24]. The processing of such large and more complex MRI datasets has rendered for clinicians as discouraging and difficult process, who can obtain valuable information manually [25]. This manual assessment is also time consuming and error prone due to the various inter- or intrinsic variability analysis carried out by the operator. These problems require new developments in computerized techniques for better diagnosis and subsequent research in brain MRI data evaluation.

Features and attributes of interest, if carefully selected, are exemplary to the maximum detail that the image can provide to demonstrate the lesion in its entirety [23]. Functional derivative methodologies analyses items plus images to obtain the most influential characteristics indicative of divergent object classes [24]. Features are used to assign them to their specified classes as ingredients for classifiers [2]. The goal of the irrelevant feature removal process is to minimize the initial data by measuring unique properties or features separating one input design from another.

Convolution is a two-function operation of a genuinely valued statement. Initially, from an Image Set IS, an MRI image is considered and from the image, pixels are extracted. If you use the data of a double-dimensional image $\mathrm{I}_{\mathrm{g}}$ and a twodimensional kernel $\mathrm{K}$ for storing the pixel representation is calculated as:

$$
\operatorname{IS}\left((\operatorname{Ig} * K)(x, y)=\sum_{x} \cdot \sum_{y} \operatorname{Ig}(x, y)+K(i-T 1, j\right.
$$

where, $\mathrm{T} 1$ and $\mathrm{t} 2$ are Threshold values indicating the edges of the images in $\mathrm{x}$ and $\mathrm{y}$ axis and $\mathrm{I}$ and $\mathrm{j}$ are extracted adjacent pixels. The discrete convolution DC operator $*$ on input image $\mathrm{I}_{\mathrm{g}}$ and considered kernel filter $\mathrm{K}$ can be defined as the following:

$$
\begin{aligned}
(I g * K)(D C)= & \sum_{M} \operatorname{Ig}(i, i+1)+\operatorname{Ig}(j, j+1) \\
& -K(i, j)+\theta
\end{aligned}
$$

where, $\theta$ is the angle of the image considered. Decision Tree is a simple algorithm that can be represented in a tree-like diagram where the classification process is performed in each node by a number of groups using a predefined criterion. This approach hierarchically divides the data into smaller subsets. These requirements can be estimated during learning by maximizing the knowledge $\mathrm{K}_{\mathrm{n}}$ gain in a given node, which is defined as reducing uncertainty after dividing the training data. The following equation is widely used for dividing the training and testing data:

$$
I g=H(S)-\sum_{i \in L, R} \frac{\left|S^{i}\right|}{|S|} H\left(S^{i}\right) * \mathrm{Kn}+\sum_{i, j} \frac{\left|I g^{i}\right|}{|I S|}
$$

Here $\mathrm{H}$ is the set of relevant features, when individual neuron layers are stacked and outputs from one to another are connected, a multi-layer network is established that is represented as

$$
M \ln i=f\left(\sum_{j=1}^{N} H(S) * I s_{j} w_{i}\right)
$$

Here is the Image set considered. Twenty features of the text such as radius_mean, texture_mean, perimeter_mean, area_mean, fractal_dimension_mean, smoothness_se etc., have been separated and isolated using the normal neural surface and separation from the specific MRI images of the brain. The extracted characteristics are then listed below. The difference between darkest and luminous pixels of the MRI image is important for studying the area calculated as follows,

$$
\begin{gathered}
\text { contrast }=\sum_{i, j=0}^{n-1} P_{i, j}(i-j)^{2} \\
\text { Dissimilarity }=\sum_{i, j=0}^{n-1}|i-j| P(i, j) \\
\text { Entropy }=\sum_{i, j=0}^{n-1}-\ln \left(P_{i j}\right) P_{i j}
\end{gathered}
$$

Here $\mathrm{P}$ is the pixel considered from the image and its relevant features are maintained as a set. The homogeneity is used to measure compactness of pixels for identification of its relevancy to identify the tumor in image is estimated using.

$$
H G(I S(W i))=\sum_{i, j=0}^{n-1} \frac{P(i, j)+\sum_{i, j=0}^{n-1} P_{i, j}(i-j)^{2}}{(i-j)^{2}+T}
$$

Here $\mathrm{T}$ is the Threshold limit for calculating homogeneity and the optimal threshold OT is a threshold with the following property:

$$
\sigma_{B}^{2}\left(O T^{*}\right)=\max _{1 \leq k \leq L} \sigma_{B}^{2}(O T)+\sum_{j=1}^{N} H G(i)+H(S)_{i}
$$


A convolutionary layer is the critical element of the architecture. One of the network parameters to be specified before training is the number and the size of kernels of a given layer. This number can differ significantly based on the application, the complexity of the problem and the image resolution. Filter kernels are normally set up randomly and modified by the back-propagation algorithm during the training process. The outputs of convolutionary layers are also known as characteristics or maps, as convolutionary filters are indeed extractors. The distinction between traditional extractors and practical extractors used in $\mathrm{CNN}$ is that they are directly learned from the data rather than produced from hand. The feature extractors are represented as

$F e K_{j}^{l}(\mathrm{IS}(\mathrm{i}))=\left(\sum_{i \in M_{j}^{l}} \sum_{i, j} \frac{\left|I g^{i}\right|}{|I S|} * O T_{i j}^{l}+I g_{j}^{l}+k_{i j}^{l}\right)$

where, $F e K_{j}^{l}$ denotes a non-linear activation function, which is typically the sigmoid function $\left(f(x)=\left(1+e^{-x}\right)^{-1}\right)$ or the hyperbolic tangent function $\left(f(x)=\left(e^{2 x}-1\right)\left(e^{2 x}+1\right)^{-1}\right)$, $O T_{i j}^{l}$ is a bias, $I g_{j}^{l}$ denotes a selection of image feature maps, and $k_{i j}^{l}$ is a kernel used in the previous layer.

The proposed work aimed at segmenting tumors in other healthy tissues. Both the tumor and the healthy tissues considered are a binary role for segmentation, there are only two groups. The label $\mathrm{L}=\{0,1\}$, where 1 represents a tumor label and 0 corresponds to the background label is assigned to each pixel. Binary segmenting energy feature is performed as:

$$
\begin{aligned}
B s(f(\operatorname{Ig}(i)))= & \sum_{p \in Q} F_{e} K_{j}^{l}\left(H G(i)+H(S)_{i}\right) \\
& +\lambda \sum_{p q \in N} K_{p q}\left(f_{i}, f_{j}\right)
\end{aligned}
$$

where, only all the pixels in the image are set and $\lambda$ is a neighborhood system of adjacent pixels, and the neighboring pixels in a neighborhood system are pixels $(i, j)$. fi alternative to $\mathrm{w}$ denotes the pixel i mark given. The labels are assigned as

$$
V_{i, j}\left(f_{i}, f_{j}\right)=w_{i, j} \cdot \delta\left(f_{i}, f_{j}\right)+\lambda+B s(f(\operatorname{Ig}(i)))
$$

If a single neuron is used as a cost function, the weight update at the iteration $i$ can be interpreted as

$$
\begin{gathered}
w(i+1)=w(i)+\Delta w(i) \\
\Delta w(i)=-\mu \nabla \varepsilon(w(i))
\end{gathered}
$$

where, $\mu$ is the apprenticeship rate and $\mathrm{w}$ is the weights-w(i) cost feature.

As the last network layer, $\mathrm{nL}$ is specified, Output feature set Ofs of the fully connected layer that is calculated as:

$$
O f s=f s\left(W^{n L} o v^{n L-1}+b^{n L}\right)
$$

As tumor pixels constitute an extremely small part of the entire slice of the image, the segmentation of a tumor from the background is a dense prediction task that is highly unbalanced. The proposed model uses a class balanced cross-entropy loss function to deal more closely with the imbalanced class problem. All network layer parameters as $\mathrm{nL}$ is considered and the target function is calculated for tumor prediction as

$$
\begin{gathered}
T p(W)=O f s+\sum_{I g \in I S} \sum_{i \in I g} F e K_{j}^{l}+\lambda \sum_{i, j \in N} K_{i, j}+\beta+ \\
\log \operatorname{Pr}\left(y_{j}=1 \mid X ; W\right)+\sum_{j \in Y} \log \operatorname{Pr}\left(y_{n}=0 \mid X ; W\right)
\end{gathered}
$$

Loss is measured in all pixels in the $X=(x j, j=1, \ldots$,$) . The$ background pixels and tumour pixels are referred background pixels and $\mathrm{Y}$ respectively. Where $\beta$ controls the relative importance between the background pixels and tumor pixels. Wi values in proportion to number of samples in the I class can be selected inversely for the loss of data for every layer that is calculated as

$$
\begin{aligned}
F S_{\text {loss }}(i, j, n L)= & -\sum_{i}^{M} W_{i} L_{i} \log S_{n} \\
& -\sum_{i}^{M}\left(1-\mathrm{n} L_{i}\right) \log \left(1-S_{i}\right) \\
& +w(i)+\Delta w(i)
\end{aligned}
$$

The Error Calculation Rate (ER) is performed using the equation

$$
E r=\frac{1}{w * P} \sum_{i=1}^{N} T+\left(H G(i)-F S^{N}\left(W^{2}\right)\right)
$$

where, $\mathrm{W}$ is the weight of the pixel $\mathrm{P}, \mathrm{T}$ is the threshold value for error rate calculation.

\section{RESULTS}

The proposed work seeks to examine the automatic segmentation of the brain tumor by combining convolutionary neural networks with machine learning in order to automatically achieve tumor segmentation. The proposed model is implemented using python and simulated in ANACONDA. The detection of brain tumors is a difficult and sensitive task involving the classifier 's experience. The use of Convolutional Neural Network (CNN) system to classify the brain tumor type is presented in this work. Relevant features are extracted from images and by using $\mathrm{CNN}$ with machine learning technique, tumor can be recognized. $\mathrm{CNN}$ can reduce the cost and increase the performance of brain tumor detection. The proposed work is compared to the traditional methods and the results show that the proposed method is effective in detecting tumors. The proposed Group based Classifier for Brain Tumor Recognition (GbCBTD) model is compared with the traditional models in terms of image segmentation accuracy, classification accuracy, feature extraction time, true positive rate, Tumor detection Accuracy. The proposed model is compared with the traditional deep convolutional neural network fusion support vector machine algorithm (DCNN-FSVM) and the results are depicted. Figure 2 depicts the image segmentation accuracy.

The Precision, Recall and F1 score of the proposed and traditional models are depicted in Table 1. 
Table 1. Precision, recall and F1 score values

\begin{tabular}{ccccccc}
\hline & \multicolumn{2}{c}{ Precision } & \multicolumn{2}{c}{ Recall } & \multicolumn{2}{c}{ F1 Score } \\
\hline & GbCBTD & DCNN-F-SVM & GbCBTD & DCNN-F-SVM & GbCBTD & DCNN-F-SVM \\
& Model & Model & Model & Model & Model & Model \\
Micro Average & 95 & 74 & 95 & 81 & 95 & 79 \\
Macro Average & 95 & 73 & 94 & 82 & 94 & 80 \\
Weighted Average & 95 & 74 & 95 & 82 & 95 & 80 \\
\hline
\end{tabular}

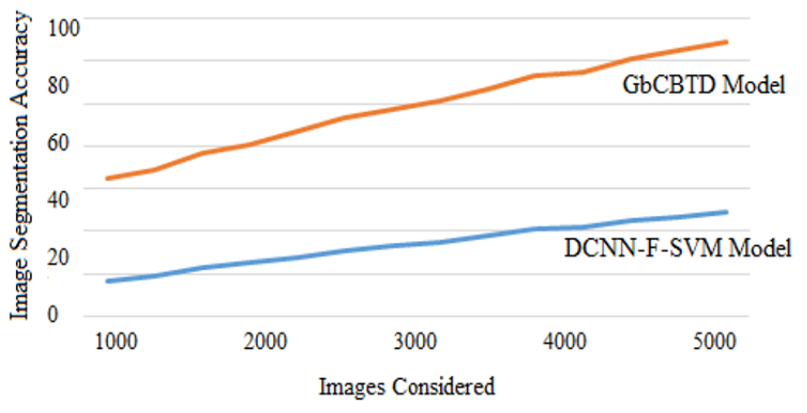

Figure 2. Image segmentation accuracy

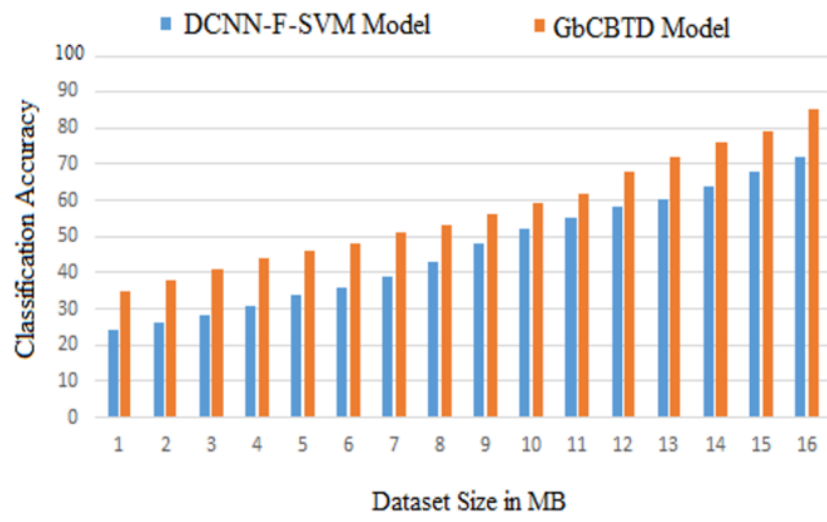

Figure 3. Classification accuracy

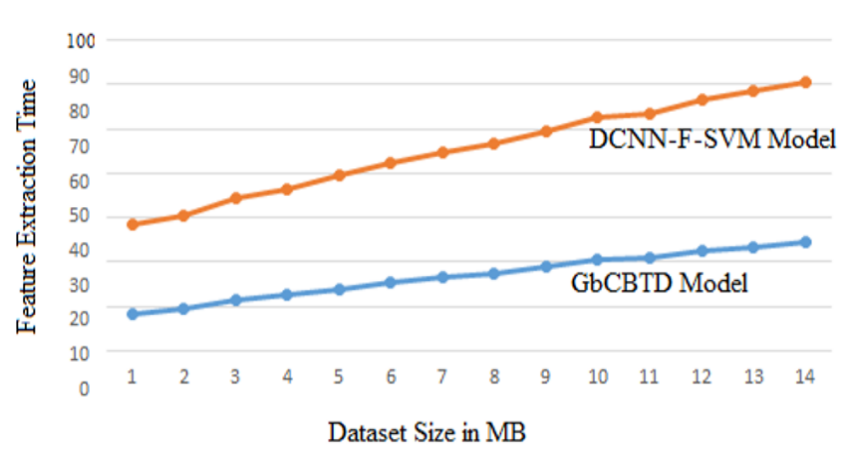

Figure 4. Feature extraction time

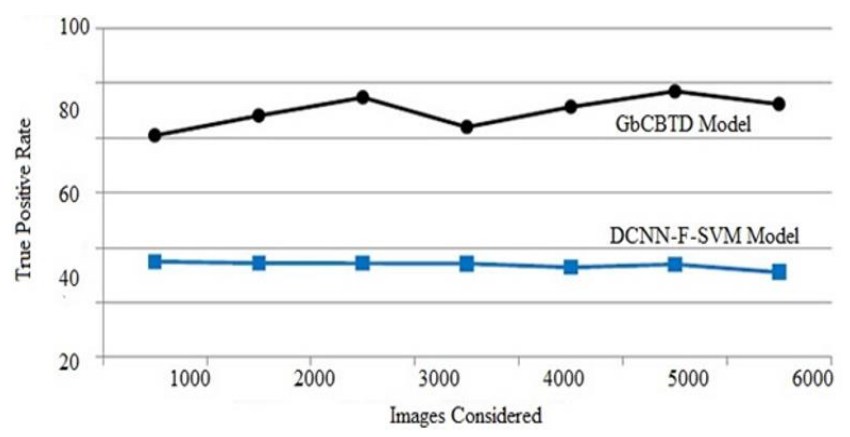

Figure 5. True positive rate
Classification is performed on the dataset for grouping related data as a group and then identification process will be performed on that grouped data. Figure 3 depicts the classification accuracy. The result show that the proposed model classification accuracy is more when compared to traditional models.

Feature Extraction is performed for considering only relevant features from the dataset. Based on the features selected, tumor identification process is applied so that tumor can be detected accurately. Figure 4 depicts the feature extraction time levels. The proposed model takes less time for extracting feature extraction when compared to existing model.

The True positive rate of the proposed and existing models is depicted in Figure 5. The proposed model has high true positive rate that represents that the tumor prediction rate is high than the existing method.

The tumor detection rate of the proposed and existing models is depicted in Figure 6. The tumor detection accuracy of the proposed model is high when compared to traditional model.

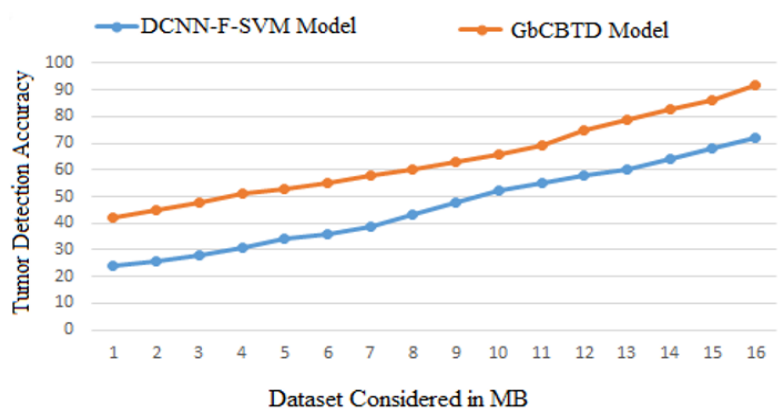

Figure 6. Tumor detection accuracy

To identify a tumor in the brain, the image pixel analysis should be done. The actual tumor cell identification calculation in the segmented image is performed and the results show that the proposed model tumor cell identification calculation time is less when compared to traditional model. Figure 7 depicts the tumor cell identification calculation time levels.

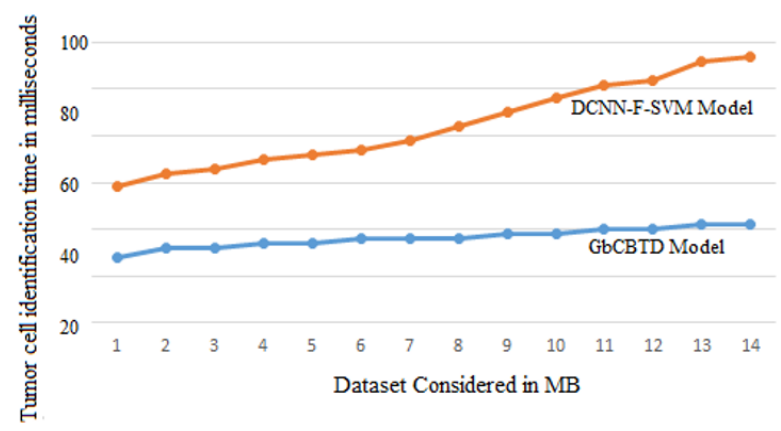

Figure 7. Tumor cell identification calculation time levels 
The operational cost of the proposed model is less when compared to the traditional methods. The results in Figure 8 indicates the operational cost levels of the proposed and traditional methods.

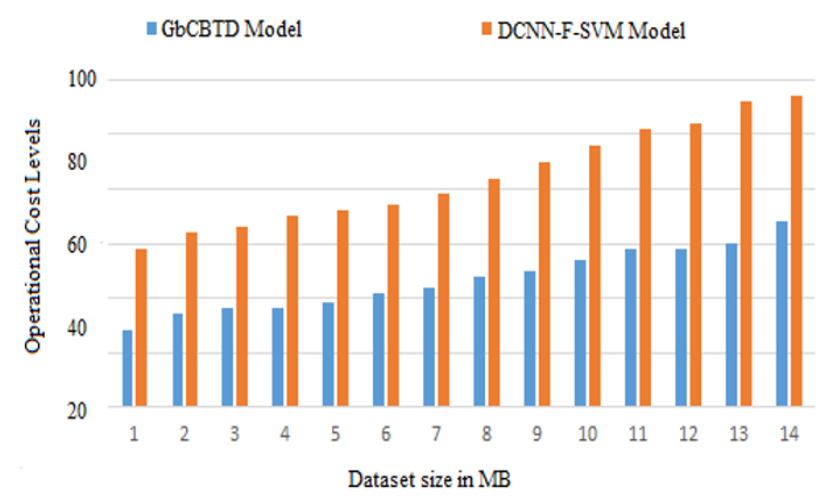

Figure 8. Oprational cost levels

\section{CONCLUSION}

Brain Tumor is the most dangerous disease that causes deaths to majority of the effected people. In the proposed work an Efficient Group Based Classifier for Brain Tumor Detection using Machine Learning Technique is introduced for effective segmentation procedure that combines convolution neural networks with machine learning. Initially the brain imager is segmented into smaller parts and then clustering is applied with the image by extracting relevant features from the segmented image. Since the tumour pixels account for a very small amount of the whole slice image, it is a highly unbalanced dense prediction task to segment tumours from the background. The loss function has been used to take into account the discrepancy of training results. The results of overlapping pixels are combined to achieve segmentation on an entire brain segment. The proposed model considers MRI image and perform segmentation on it by extracting relevant features that are useful for the identification of tumor cells after applying segmentation. The proposed model is compared with the traditional methods and the accuracy of the proposed model is high when compared to existing methods. In future, the features considered can be reduced and the hidden layers can be improved for better image segmentation. For the features extracted weights can also be assigned for considering the priority features only for the tumor prediction.

\section{REFERENCES}

[1] Qian, P., Zheng, J., Zheng, Q., Liu, Y., Wang, T., Al Helo, R. (2020). Transforming UTE-mDixon MR abdomenpelvis images into $\mathrm{CT}$ by jointly leveraging prior knowledge and partial supervision. IEEE/ACM Transactions on Computational Biology and Bioinformatics, pp. https://doi.org/10.1109/TCBB.2020.2979841

[2] Havaei, M., Davy, A., Warde-Farley, D., Biard, A., Courville, A., Bengio, Y., Courville, A., Beng, Y., Pal, C., Jodoin, P., Larochelle, H. (2017). Brain tumor segmentation with deep neural networks. Medical Image Analysis, 35:

$18-31$.
[3] Menze, B.H., Jakab, A., Bauer, S., et al. (2014). The multimodal brain tumor image segmentation benchmark (BRATS). IEEE Transactions on Medical Imaging, 34(10):

1993-2024. https://doi.org/10.1109/TMI.2014.2377694

[4] Chaurasia, A., Culurciello, E. (2017). Linknet: Exploiting encoder representations for efficient semantic segmentation. In 2017 IEEE Visual Communications and Image Processing (VCIP), pp. 1-4. https://doi.org/10.1109/VCIP.2017.8305148

[5] Bauer, S., Nolte, L.P., Reyes, M. (2011). Fully automatic segmentation of brain tumor images using support vector machine classification in combination with hierarchical conditional random field regularization. In International Conference on Medical Image Computing and Computer-Assisted Intervention, pp. 354-361. https://doi.org/10.1007/978-3-642-23626-6_44

[6] Qian, P., Chen, Y., Kuo, J.W., Zhang, Y.D., Jiang, Y., Zhao, K. (2019). mDixon-based synthetic CT generation for PET attenuation correction on abdomen and pelvis jointly using transfer fuzzy clustering and active learning-based classification. IEEE Transactions on Medical Imaging, 39(4): 819-832. https://doi.org/10.1109/TMI.2019.2935916

[7] Jiang, Y., Zhao, K., Xia, K., Xue, J., Zhou, L., Ding, Y., Qian, P. (2019). A novel distributed multitask fuzzy clustering algorithm for automatic MR brain image segmentation. Journal of Medical Systems, 43(5): 118. https://doi.org/10.1007/s10916-019-1245-1

[8] Qian, P., Xu, K., Wang, T., Zheng, Q., Yang, H., Baydoun, A., Zhu, J.Q., Traughber, B., Muzic, R.F. (2020). Estimating CT from MR Abdominal Images Using Novel Generative Adversarial Networks. Journal of Grid Computing, 18: 211-226. https://doi.org/10.1007/s10723-020-09513-3

[9] Xia, K., Zhong, X., Zhang, L., Wang, J. (2019). Optimization of diagnosis and treatment of chronic diseases based on association analysis under the background of regional integration. Journal of Medical Systems, 43(3): 46. https://doi.org/10.1007/s10916-0191169-9

[10] Qian, P., Xi, C., Xu, M., Jiang, Y., Su, K.H., Wang, S., Muzic Jr, R.F. (2018). SSC-EKE: Semi-supervised classification with extensive knowledge exploitation. Information Sciences, 422: 51-76. https://doi.org/10.1016/j.ins.2017.08.093

[11] Jiang, Y., Deng, Z., Chung, F.L., Wang, G., Qian, P., Choi, K.S., Wang, S. (2016). Recognition of epileptic EEG signals using a novel multiview TSK fuzzy system. IEEE Transactions on Fuzzy Systems, 25(1): 3-20. https://doi.org/10.1109/TFUZZ.2016.2637405

[12] Qian, P., Zhou, J., Jiang, Y., Liang, F., Zhao, K., Wang, S.T., Su, K.H., Muzic, R.F. (2018). Multi-view maximum entropy clustering by jointly leveraging interview collaborations and intra-view-weighted attributes. IEEE Access, 6: 28594-28610. https://doi.org/10.1109/ACCESS.2018.2825352

[13] Jiang, Y., Wu, D., Deng, Z., Qian, P., Wang, J., Wang, G., Chung, F.L., Choi, K.S., Wang, S. (2017). Seizure classification from EEG signals using transfer learning, semi-supervised learning and TSK fuzzy system. IEEE Transactions,

pp. 2270-2284. https://doi.org/10.1109/TNSRE.2017.2748388

[14] Kadkhodaei, M., Samavi, S., Karimi, N., Mohaghegh, H., 
Soroushmehr, S.M.R., Ward, K., All, A., Najarian, K. (2016). Automatic segmentation of multimodal brain tumor images based on classification of super-voxels. In 2016 38th Annual International Conference of the IEEE Engineering in Medicine and Biology Society (EMBC), pp. $5945-5948$. https://doi.org/10.1109/EMBC.2016.7592082

[15] Jafari, M.H., Karimi, N., Nasr-Esfahani, E., Samavi, S., Soroushmehr, S.M.R., Ward, K., Najarian, K. (2016). Skin lesion segmentation in clinical images using deep learning. In 2016 23rd International Conference on Pattern Recognition (ICPR), pp. 337-342. https://doi.org/10.1109/ICPR.2016.7899656

[16] Nasr-Esfahani, M., Mohrekesh, M., Akbari, M., Soroushmehr, S.R., Nasr-Esfahani, E., Karimi, N., Samavi, S., Najarian, K. (2018). Left ventricle segmentation in cardiac MR images using fully convolutional network. In 2018 40th Annual International Conference of the IEEE Engineering in Medicine and Biology Society (EMBC), pp. 1275-1278. https://doi.org/10.1109/EMBC.2018.8512536

[17] Rafiei, S., Nasr-Esfahani, E., Najarian, K., Karimi, N., Samavi, S., Soroushmehr, S.R. (2018). Liver segmentation in CT images using three dimensional to two dimensional fully convolutional network. In 2018 25th IEEE International Conference on Image Processing (ICIP), $\mathrm{pp}$. 2067-2071 https://doi.org/10.1109/ICIP.2018.8451238

[18] Abiwinanda, N., Hanif, M., Hesaputra, S.T., Handayani, A., Mengko, T.R. (2019). Brain tumor classification using convolutional neural network. In World Congress on Medical Physics and Biomedical Engineering 2018, pp. 183-189. https://doi.org/10.1007/978-981-10-90356_33
[19] Parihar, A.S. (2017). A study on brain tumor segmentation using convolution neural network. In 2017 International Conference on Inventive Computing and Informatics (ICICI), pp. 198-201. https://doi.org/10.1109/ICICI.2017.8365336

[20] Kwon, D., Shinohara, R.T., Akbari, H., Davatzikos, C. (2014). Combining generative models for multifocal glioma segmentation and registration. In International Conference on Medical Image Computing and Computer-Assisted Intervention, pp. 763-770. https://doi.org/10.1007/978-3-319-10404-1_95

[21] Geremia, E., Menze, B.H., Ayache, N. (2012). Spatial decision forests for glioma segmentation in multichannel MR images. MICCAI Challenge on Multimodal Brain Tumor Segmentation, 57(2): 378-390. https://doi.org/10.1016/j.neuroimage.2011.03.080

[22] Le Folgoc, L., Nori, A.V., Ancha, S., Criminisi, A. (2016). Lifted auto-context forests for brain tumour segmentation. In International Workshop on Brainlesion: Glioma, Multiple Sclerosis, Stroke and Traumatic Brain Injuries, pp. 171-183. https://doi.org/10.1007/978-3319-55524-9_17

[23] Zhang, Z., Sejdić, E. (2019). Radiological images and machine learning: Trends, perspectives, and prospects. Computers in Biology and Medicine, 108(6): 354-370. https://doi.org/10.1016/j.compbiomed.2019.02.017

[24] Zikic, D., Ioannou, Y., Brown, M., Criminisi, A. (2014). Segmentation of brain tumor tissues with convolutional neural networks. Proceedings MICCAI-BRATS, pp. 3639.

[25] Sajja, T.K., Devarapalli, R.M., Kalluri, H.K. (2019). Lung cancer detection based on CT scan images by using deep transfer learning. Traitement du Signal, 36(4): 339344. https://doi.org/10.18280/ts.360406 